\title{
Foot posture in people with medial compartment knee osteoarthritis
}

\author{
Pazit Levinger ${ }^{1 *}$, Hylton B Menz ${ }^{1}$, Mohammad R Fotoohabadi ${ }^{1}$, Julian A Feller ${ }^{1}$, John R Bartlett ${ }^{2}$, Neil R Bergman²
}

\begin{abstract}
Background: Foot posture has long been considered to contribute to the development of lower limb musculoskeletal conditions as it may alter the mechanical alignment and dynamic function of the lower limb. This study compared foot posture in people with and without medial compartment knee osteoarthritis (OA) using a range of clinical foot measures. The reliability of the foot measures was also assessed.

Methods: The foot posture of 32 patients with clinically and radiographically-confirmed OA predominantly in the medial compartment of the knee and 28 asymptomatic age-matched healthy controls was investigated using the foot posture index (FPI), vertical navicular height and drop, and the arch index. Independent t tests and effect size (Cohen's d) were used to investigate the differences between the groups in the foot posture measurements.

Results: Significant differences were found between the control and the knee OA groups in relation to the FPI $(1.35 \pm 1.43$ vs. $2.46 \pm 2.18, p=0.02 ; d=0.61$, medium effect size), navicular drop ( $0.02 \pm 0.01$ vs. $0.03 \pm 0.01, p=$ $0.01 ; d=1.02$, large effect size) and the arch index (0.22 \pm 0.04 vs. $0.26 \pm 0.04, p=0.04 ; d=1.02$, large effect size). No significant difference was found for vertical navicular height ( $0.24 \pm 0.03$ vs. $0.23 \pm 0.03, p=0.54 ; d=0.04$, negligible effect size).
\end{abstract}

Conclusion: People with medial compartment knee OA exhibit a more pronated foot type compared to controls. It is therefore recommended that the assessment of patients with knee OA in clinical practice should include simple foot measures, and that the potential influence of foot structure and function on the efficacy of foot orthoses in the management of medial compartment knee OA be further investigated.

\section{Background}

Knee osteoarthritis (OA) is a common painful and chronic condition that affects a large proportion of the older population [1,2]. Knee OA may in part be due to excessive loading of the articular cartilage [3]. During walking, the forces transmitted across the knee joint are greater in the medial compartment compared to the lateral compartment [4], and increased medial compartment loading has been observed in patients with knee OA [5-8]. The mechanics of gait, in particular the knee adduction moment (the moment that tends to adduct the knee during the stance phase of walking), have been shown to be a contributing factor to the progression of medial compartment knee OA [5-7,9]. Treatment strategies for knee OA, such us foot orthoses, knee braces

\footnotetext{
* Correspondence: p.levinger@latrobe.edu.au

'Musculoskeletal Research Centre, Faculty of Health Sciences, La Trobe University. Bundoora, Victoria 3086, Australia

Full list of author information is available at the end of the article
}

and footwear, have been proposed to minimise the knee adduction moment, and consequently reduce the loading on the medial compartment [10-18].

Foot posture has long been considered to contribute to the development of a range of lower limb musculoskeletal conditions $[19,20]$ as it may alter the mechanical alignment and dynamic function of the lower limb [21]. Special attention, therefore, has been given to foot orthoses and footwear modifications as a non-operative treatment of knee OA $[13,15,18,22,23]$. However, in order to fully understand the effect of these interventions on the knee and other lower limb joints and to identify patients who are most likely to benefit from them, greater knowledge of foot structure in this population is required.

Despite the potential importance of understanding foot characteristics of people with medial compartment knee OA, few studies have examined foot posture in this population. Reilly et al [24] compared navicular

\section{Biomed Central}


height in sitting and standing in 60 people with hip OA, 60 people with knee OA and 60 controls, and found no differences between the knee OA and control groups. However, there was a significant difference in frontal plane calcaneal angle, indicating a more everted rearfoot in the knee OA group. In a subsequent study, these authors also compared foot posture index (FPI) scores between 20 people with knee OA and 20 controls, and reported a significantly higher median score in those with knee OA (7.0 versus 1.0), indicative of a more pronated foot posture [25].

A key consideration when interpreting these findings is the reliability of the foot posture measures. Previous studies have indicated that frontal plane calcaneal measures have questionable reliability [26], while FPI reliability is moderate to good, depending on the clinical experience of the assessor [27]. Given the questionable reliability reported for some of the foot measures and the expertise required to take these measures [26-30], using an objective measure that does not require any subjective interpretation may be important to include as part of foot posture assessment. However, evaluation of such a measure in people with knee OA has not previously been investigated. The primary aim of this study therefore was to investigate foot type in people with and without medial compartment knee OA using a range of clinical foot measures, including a measure (the arch index) that requires no clinical expertise or subjective interpretation. A secondary aim was to determine the reliability of the foot posture measurements.

\section{Methods}

Two groups participated in the study: a knee OA group and an age-matched asymptomatic control group. The OA group included 32 participants diagnosed with predominantly medial compartment $\mathrm{OA}$, determined by radiographic assessment. The severity of knee OA was based on the loss of joint space determined by an orthopaedic surgeon from radiographic images [31] and was graded as follows: 1- less than a half of joint space loss (mild), 2 - more than a half of joint space loss; bone on bone (moderate) and 3 - bone deformity/loss of bone (severe). Each compartment of the knee joint (medial compartment, lateral compartment and patellofemoral compartment) was graded and participants with predominantly medial compartment OA (severity grade 2-3) were included in the study. Participants from the OA group were included if they were able to walk independently and were excluded if they had uncontrolled systemic disease and or a pre-existing neurological or other orthopaedic condition that affected their walking. Participants from the OA group were recruited from the La Trobe University Medical Centre, the Warringal Private Medical Centre and through advertisements in local newspapers. The control group consisted of 28 asymptomatic participants with no clinical diagnosis of OA, rheumatoid arthritis or history of knee trauma or pain. Participants from the control group were recruited from retirement villages in northern Melbourne and through advertisements in local newspapers. Ethics approval was obtained from the Faculty of Health Sciences Human Ethics Committee, La Trobe University. All participants were informed about the nature of the study and signed a consent form prior to participation.

\section{Procedure}

All participants attended the gait laboratory at La Trobe University for a single session, and 23 participants from the control group attended on two occasions to assess the reliability of the foot measurements. All foot measurements were assessed by the same examiner (PL) with previous experience in taking these measures [27]. Participants' body mass, height and truncated foot length were recorded. The symptomatic leg (or the most symptomatic leg in a case of bilateral involvement) in the OA group and the same corresponding leg of each peer control matched for age were assessed.

\section{Foot posture measurements}

The foot posture measurements included the foot posture index (FPI), navicular height, navicular drop and the arch index. The FPI is a 6-item foot posture assessment with the subject standing relaxed in a bipedal position [29]. The 6 items of the FPI include talar head palpation, curves above and below the lateral malleoli, calcaneal angle, talonavicular bulge, medial longitudinal arch and forefoot to rearfoot alignment. Each item was scored on a 5-point scale between -2 and +2 and provides a total sum of all items between -12 (highly supinated) and +12 (highly pronated). The raw FPI scores were converted to Rash transformed scores to allow the scores to be used as interval data [32]. The transformed FPI values were used for the analysis.

Navicular height and navicular drop measurements were taken in subtalar joint neutral (STJN) position and in relaxed standing posture using a business card as described previously [33] and with the aid of a rightangled metal bracket for stabilising the card [27]. STJN was defined as the position of the foot when the talar head could be palpated just anterior to the ankle mortise with equal prominence both medially and laterally. The position of the subtalar joint in neutral was maintained and the vertical height of the navicular was marked on the business card. The participants were then asked to relax and the vertical height of the navicular was marked on the card. Navicular drop was measured as the difference between the STJN and relaxed stance of the navicular height (see Figure 1). Both 


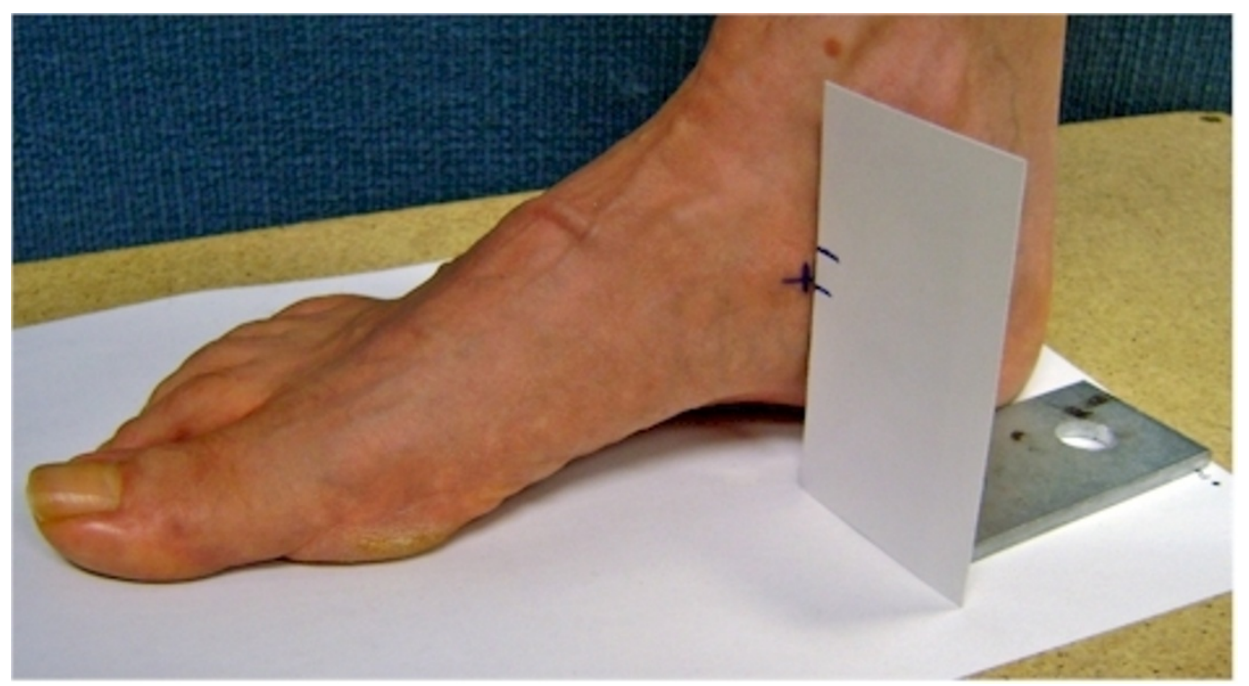

Figure 1 Navicular height and drop measurement.

measures were normalised to each participant's truncated foot length. Truncated foot length was measured from the most posterior aspect of the calcaneus to the first metatarsophalangeal joint. Truncated foot length was used for normalisation due to the potential presence of toe deformity in older people which can affect the foot length value [34].

The arch index was measured with the participant standing on a carbon paper imprint material in relaxed bipedal stance. A static footprint was obtained and was divided to three equal sections. The arch index was then calculated as the ratio of the middle section to the entire footprint area using a computer graphics tablet (Wacom Technology Corporation, Vancouver, Canada). Higher values of the arch index indicate a flatter (more pronated) foot [35]. See Figure 2.

\section{Statistical analysis}

All analyses were performed using SPSS 17.0 for Windows (SPSS Inc., Chicago IL, USA). The intra-rater reliability of the foot posture measurements was evaluated using intraclass correlation coefficients $\left(\mathrm{ICCs}_{3,1}\right)$, 95\% limits of agreement and coefficient of variation [36]. ICCs above 0.90 were considered excellent, 0.75 0.90 considered good, $0.50-0.75$ considered moderate and ICC below 0.50 considered poor [37]. Differences between the groups were assessed using independent samples t-tests for continuously scored variables and chi-squared statistics for categorical variables. The magnitude of the differences in continuously-scored variables between the groups was assessed using Cohen's $d$, with the following cut-offs applied to aid interpretation: $<0.15$ - negligible effect, $\geq 0.15$ to $<0.40$ - small effect, $\geq 0.40$ to $<0.75$ - medium effect, $\geq 0.75$ to $<1.10$ - large effect, $\geq 1.10$ to $<1.45$ - very large effect, and $>1.45$ huge effect [38]. To explore the potential correlation between body weight and the foot posture measures, Pearson's correlation coefficient was used. Where significant correlations were found, bodyweight was used as a covariate for that particular foot posture measure.

\section{Results}

The demographic characteristics of both groups are summarised in Table 1 . The participants' age and height were similar between the groups, although the knee OA group had a significantly greater body weight and body mass index. The ICCs for the foot measures ranged from moderate to excellent. Navicular height and drop showed ICC $=0.86$ and ICC $=0.56$, respectively, with FPI and arch index having ICC $=0.91$ and ICC $=0.93$, respectively. Similarly, low coefficients of variation were found for the FPI, navicular height and arch index (Table 2).

A significant correlation was found between body weight and the arch index $(r=0.44, p<0.001)$ with no significant correlation between body weight and FPI ( $r=$ $0.22, p=0.09)$, navicular height $(r=0.008, p=0.94)$ or navicular drop $(r=0.20, p=0.12)$. Body weight was therefore entered as a covariate for the comparison of the arch index between the groups.

Significant differences were found between the groups for three foot measures, with the knee OA group exhibiting a more pronated foot compared to the control group for the FPI ( $2.46 \pm 2.18$ vs $1.35 \pm 1.43$.; $p=0.02 ; d=0.61$, medium effect size), navicular drop (0.03 \pm 0.01 vs $0.02 \pm$ $0.01 ; p=0.01 ; d=1.02$, large effect size) and arch index $(0.26 \pm 0.04$ vs $0.22 \pm 0.04 ; p=0.04 ; d=1.02$, large effect size) as indicated in Figure 3. No significant difference 

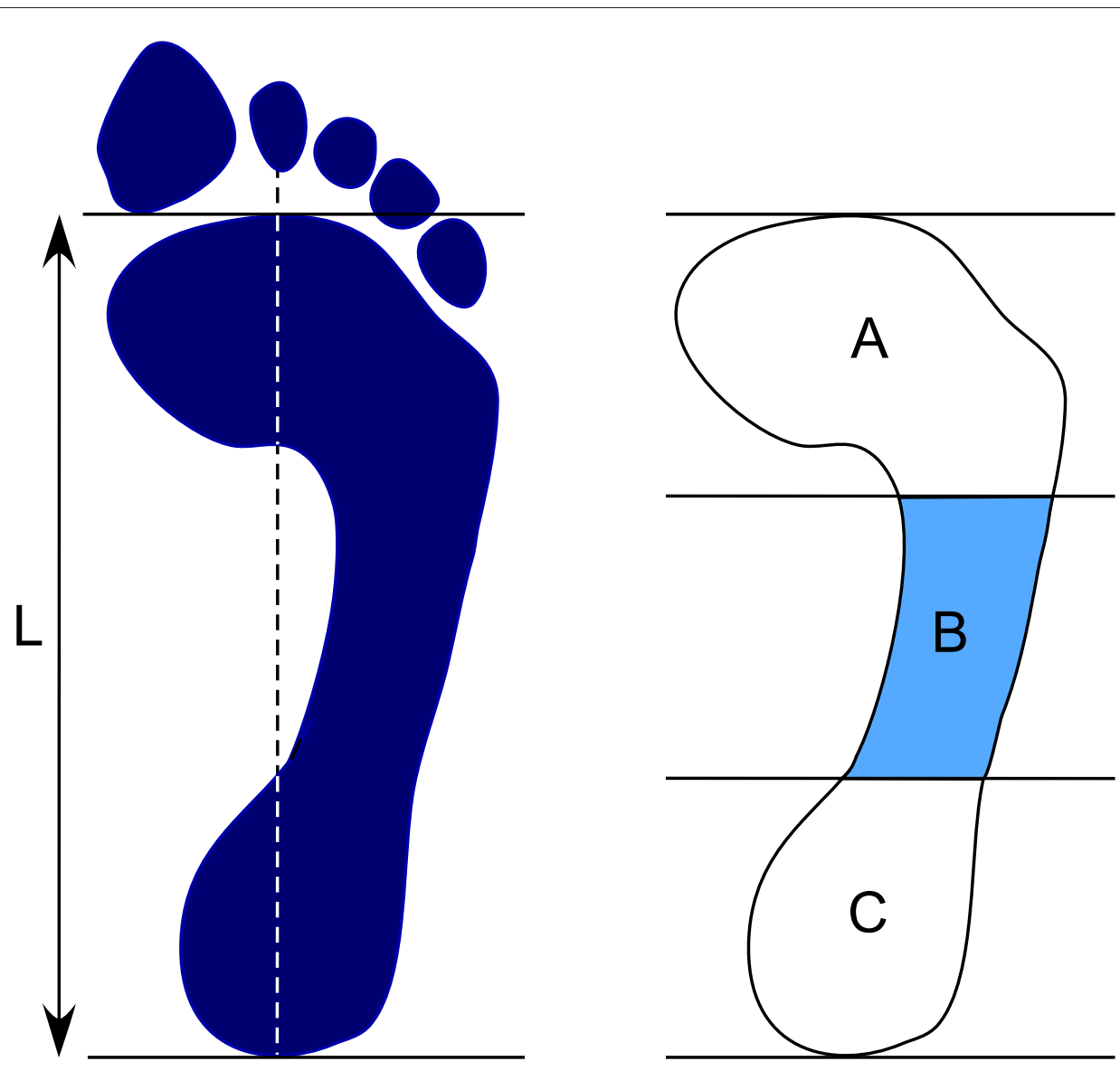

Figure 2 Calculation of the Al. The truncated length of the footprint $(\mathrm{L})$ is divided into equal thirds. The $\mathrm{Al}$ is then calculated as the area of the middle third of the footprint divided by the entire footprint area $(A I=B /[A+B+C]$ ).

was found between the groups for navicular height (Table 3).

\section{Discussion}

Foot posture has long been considered to influence the mechanical alignment and dynamic function of the lower limb and may therefore be related to the development of lower limb musculoskeletal conditions. Subsequently, several recent studies have drawn attention to the potential benefits of foot orthoses in reducing the load on the knee, particularly the knee adduction moment $[13,15,18,22,23]$. Assessing foot characteristics of people with medial compartment OA may therefore advance our understanding of the potential role of foot orthoses and footwear modifications on lower limb alignment and function.

In this study, we investigated foot characteristics of people with medial compartment knee OA using several foot measures. The OA group exhibited a more pronated foot type compared to the control group, as indicated by the three foot measures: FPI, navicular drop and arch index, with medium to large effect sizes. Similar findings were reported by Reilly and colleagues for people with severe knee medial compartment OA using

Table 1 Participants' demographic characteristics

\begin{tabular}{|c|c|c|c|}
\hline Parameters & Control group $(n=28)$ & Knee OA group $(n=32)$ & $p$ value \\
\hline Age - yr & $65.22 \pm 11.41$ & $65.84 \pm 7.57$ & 0.810 \\
\hline Female - n (\%) & $15(54)$ & $16(46)$ & 0.210 \\
\hline Height - cm & $168.61 \pm 10.64$ & $168.83 \pm 9.54$ & 0.932 \\
\hline Body weight - kg & $73.12 \pm 15.49$ & $85.13 \pm 13.67$ & $0.003^{*}$ \\
\hline Body mass index $-\mathrm{kg} / \mathrm{m}^{2}$ & $25.56 \pm 3.95$ & $29.97 \pm 5.26$ & $0.001^{*}$ \\
\hline
\end{tabular}

Values are reported as mean \pm SD unless otherwise noted.* significant at $p<0.05$. 
Table 2 Reliability of the foot posture measurements.

\begin{tabular}{lccccc}
\hline Measures & Session $\mathbf{1}$ mean \pm SD & Session $\mathbf{2}$ mean \pm SD & ICC $_{\mathbf{3 , 1}}$ (95\% CI) & 95\% LoA & CV (\%) \\
\hline Foot posture indext & $1.33 \pm 1.47$ & $1.46 \pm 1.33$ & 0.91 (0.82 to 0.96$)$ & 1.44 to -1.88 & 24 \\
Navicular height & $0.24 \pm 0.03$ & $0.23 \pm 0.03$ & 0.86 (0.71 to 0.94$)$ & 0.04 to -0.03 & 6 \\
Navicular drop & $0.01 \pm 0.01$ & $0.01 \pm 0.01$ & 0.56 (0.20 to 0.79$)$ & 0.02 to -0.02 & 38 \\
Arch index & $0.21 \pm 0.04$ & $0.21 \pm 0.04$ & 0.93 (0.84 to 0.97) & 0.03 to -0.03 & 5 \\
\hline
\end{tabular}

NB: ICC - intraclass correlation coefficient; LoA - 95\% limit of agreement; CV - coefficient of variation. † Rasch transformed FPI scores

several foot measures, including the FPI $[24,25]$. However, we found no significant difference in navicular height between the groups, which is also in agreement with Reilly and colleagues [24].

Whether pronated foot posture is a risk factor for, or a consequence of, medial compartment knee OA cannot be determined from cross-sectional studies such as ours. People with medial compartment knee OA often display genu varum malalignment of the knee, which has been shown to increase the risk of development and progression of knee OA [39,40]. Genu varum malalignment of the knee may lead to compensatory foot pronation to enable the foot to be plantigrade when weightbearing [41]. In a recent study, a simulated genu varum walking pattern was found to increase the subtalar joint pronation moment, suggesting that frontal plane angular deformities of the knee can alter the kinetic and kinematics of the foot during gait [42]. Increased foot pronation could potentially reduce the adduction moment by shifting the centre of pressure laterally, so it is possible that the foot adapts to reduce the load on the medial compartment. However, the degree of genu varum that can be compensated by foot pronation depends on the available range of motion of the ankle, subtalar and midtarsal joints [43]. Due to the potential effect of foot alignment on the loading axis of the lower limb, a longitudinal investigation is required to better understand the contribution of foot structure and function to the development of medial compartment knee OA.

The findings reported here may have implications for orthotic and footwear interventions that are commonly suggested for the management of knee OA. In particular, laterally wedged insoles have been proposed for people with medial compartment knee OA, as they have

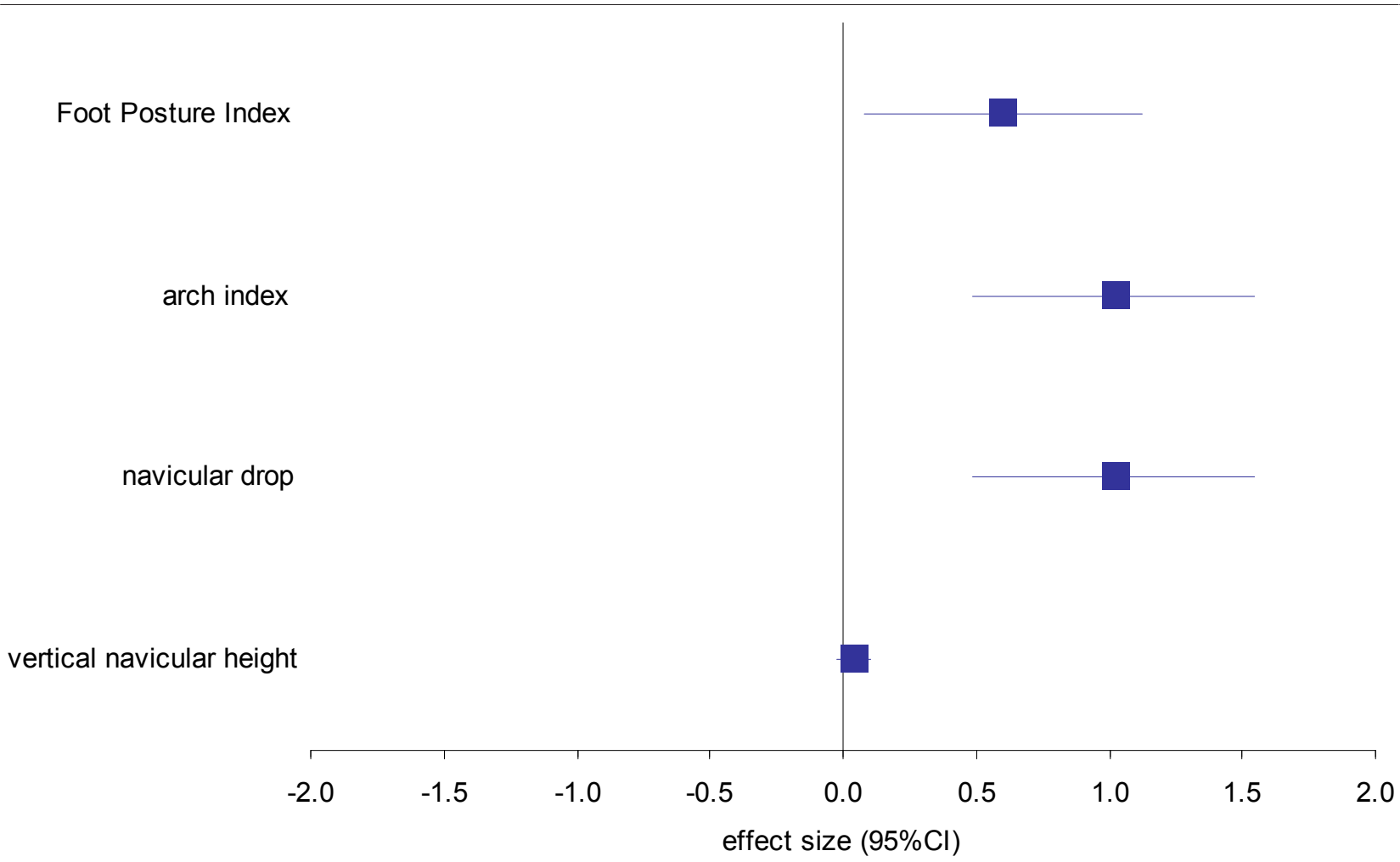

Figure 3 Effect sizes and $95 \%$ confidence intervals for the difference in foot posture variables between the control and knee OA groups. Positive values indicate larger scores in the knee OA group, negative values indicate larger scores in the control group. 
Table 3 Differences in foot posture measurements between the groups.

\begin{tabular}{lcccc}
\hline Measure & Control $(\mathbf{n}=\mathbf{2 8})$ & Knee OA $(\mathbf{n}=\mathbf{3 2})$ & p value & Effect size (Cohen's d) \\
\hline Foot posture indext & $1.35 \pm 1.43$ & $2.46 \pm 2.18$ & $0.022^{*}$ & $\mathrm{~d}=0.61$ (medium) \\
Navicular height & $0.24 \pm 0.03$ & $0.23 \pm 0.03$ & 0.542 & $\mathrm{~d}=0.04$ (negligible) \\
Navicular drop & $0.02 \pm 0.01$ & $0.03 \pm 0.01$ & $0.019^{*}$ & $\mathrm{~d}=1.02$ (large) \\
Arch index & $0.22 \pm 0.04$ & $0.26 \pm 0.04$ & $0.040^{*}$ & $\mathrm{~d}=1.02$ (large) \\
\hline
\end{tabular}

Values are reported as mean \pm SD.* significant at $p<0.05$. $\dagger$ Rasch transformed FPI scores

been shown to reduce the knee adduction moment and reduce symptoms $[12,13,18,22,23]$. However, laterally wedged insoles can alter foot motion, specifically increasing rearfoot pronation $[44,45]$. Accentuation of rearfoot pronation in already pronated feet could potentially result in detrimental changes to lower limb kinematics, and consequently lead to the development of musculoskeletal problems in other regions. Interestingly, studies have shown that the biomechanical effects of laterally wedged insoles are inconsistent, with some participants exhibiting increases in the knee adduction moment $[46,47]$. Furthermore, Nakajima et al [14] have recently reported that the addition of an arch support to laterally wedged insoles maintains normal rearfoot motion while also enhancing the ability of the insole to reduce the knee adduction moment. These findings indicate that the biomechanical effects of laterally wedged insoles may be influenced by individual variation in foot function. As such, there may be a need to include foot posture screening to appropriately identify those who are most likely to benefit from laterally wedged insoles, in order to guide the selection of modifications such as the addition of arch supports.

The reliability of foot measures has been widely reported in a range of populations [26-30]. In the present study, good to excellent intrarater reliability was found for the navicular height, arch index and FPI which was comparable to previous studies assessing intrarater reliability $[27,48,49]$ where the examiners had experience in taking foot measures. In contrast, the reliability of navicular height was only moderate, which was similar to the reliability reported by Evans et al for an adult population [49]. Measuring navicular drop involves placing the subtalar joint in neutral which requires clinical experience in order to achieve an acceptable level of reliability. However, the examiner in our study had previous experience in taking foot measures with good intrarater and interrater reliability, as we have previously reported in a younger population [27]. We therefore believe that the moderate reliability may be related to the age of our sample. Placing the subtalar joint in neutral during standing may be less reliable in older people as it requires active involvement of the participant [50] which can be challenging due to difficulty in maintaining balance.
The arch index is a reliable tool that quantifies foot characteristics based on a static footprint, and as such does not rely on the clinical experience of the examiner. The arch index however, has not been assessed previously in people with knee OA. Our results indicate that the arch index demonstrates excellent reliability, and can detect differences in foot posture between people with and without medial compartment knee OA. Importantly, the differences between the groups persisted after adjusting for bodyweight, which addresses previous concerns that the arch index may be a measure of 'fat' rather than 'flat' feet [51]. These findings suggest that the arch index may have some clinical utility in the assessment of patients with knee OA.

\section{Conclusion}

People with medial compartment knee OA exhibit a more pronated foot type compared to controls, as indicated by the FPI, navicular drop and arch index. It is therefore recommended that the assessment of patients with knee OA in clinical practice should include simple foot posture measures, and that the potential influence of foot structure and function on the efficacy of foot orthoses in the management of medial compartment knee OA be further investigated.

\section{Acknowledgements}

This study was funded by the Clive and Vera Ramaciotti Foundation and the Arthritis Foundation of Australia. HBM is currently a National Health and Medical Research Council fellow (Clinical Career Development Award, ID: 433049). We would like to thank Marg Perrott for her assistance in data collection.

\section{Author details}

${ }^{1}$ Musculoskeletal Research Centre, Faculty of Health Sciences, La Trobe University. Bundoora, Victoria 3086, Australia. ${ }^{2}$ Warringal Medical Centre, Heidelberg, Victoria 3084, Australia.

\section{Authors' contributions}

PL: designed and managed the study, collected and analysed the data drafted the manuscript. HBM: participated in the study design and assisted in the statistical analysis and data interpretation, helped to draft the manuscript. RF: assisted in data collection, data analysis. JF, JB and NB have assisted in patient recruitment, grading $x$-ray severity and drafting the manuscript. PL, HBM and JF obtained the funding. All authors have read and approved the final version.

\section{Competing interests}

HBM is Editor-in-Chief of the Journal of Foot and Ankle Research. It is journal policy that editors are removed from the peer review and editorial decision making processes for papers they have co-authored. 
Received: 13 August 2010 Accepted: 16 December 2010

Published: 16 December 2010

\section{References}

1. Access Economics: Painful realities: the economic impact of arthritis in Australia in 2007. Sydney: Arthritis Australia; 2007.

2. Woolf $A D$, Pfleger $B$ : Burden of major musculoskeletal conditions. Bull World Health Organ 2003, 81:646-656.

3. Roemhildt ML, Coughlin KM, Peura GD, Badger GJ, Churchill D, Fleming BC, Beynnon BD: Effects of increased chronic loading on articular cartilage material properties in the Lapine tibio-femoral joint. J Biomech 2010, 43:2301-2308

4. Schipplein OD, Andriacchi TP: Interaction between active and passive knee stabilizers during level walking. J Orthop Res 1991, 9:113-119.

5. Miyazaki T, Wada M, Kawahara H, Baba H, Shimada S: Dynamic load at baseline can predict radiographic disease progression in medial compartment knee. Ann Rheum Dis 2002, 61:617-622.

6. Lynn S, Reid S, Costigan P: The influence of gait pattern on signs of knee osteoarthritis in older adults over a 5-11 year follow-up period: a case study analysis. Knee 2007, 14:22-28.

7. Baliunas AJ, Hurwitz DE, Ryals AB, Karrar A, Case JP, Block JA, Andriacchi TP: Increased knee joint loads during walking are present in subjects with knee osteoarthritis. Osteoarthritis Cartilage 2002, 10:573-579.

8. Mundermann A, Dyrby CO, Andriacchi TP: Secondary gait changes in patients with medial compartment knee osteoarthritis: increased load at the ankle, knee, and hip during walking. Arthritis Rheum 2005, 52:2835-2844.

9. Andriacchi TP: Dynamics of knee malalignment. Orthop Clin North Am 1994, 25:395-403.

10. Erhart JC, Mundermann A, Elspas B, Giori NJ, Andriacchi TP: A variablestiffness shoe lowers the knee adduction moment in subjects with symptoms of medial compartment knee osteoarthritis. J Biomech 2008, 41:2720-2725.

11. Shakoor N, Lidtke RH, Sengupta M, Fogg LF, Block JA: Effects of specialized footwear on joint loads in osteoarthritis of the knee. Arthritis Rheum 2008, 59:1214-1220.

12. Crenshaw SJ, Pollo FE, Calton EF: Effects of lateral-wedged insoles on kinetics at the knee. Clin Orthop Relat Res 2000, 185-192.

13. Shimada S, Kobayashi S, Wada M, Uchida K, Sasaki S, Kawahara H, Yayama T, Kitade I, Kamei K, Kubota M, Baba H: Effects of disease severity on response to lateral wedged shoe insole for medial compartment knee osteoarthritis. Arch Phys Med Rehabil 2006, 87:1436-1441.

14. Nakajima K, Kakihana W, Nakagawa T, Mitomi H, Hikita A, Suzuki R, Akai M, Iwaya T, Nakamura K, Fukui N: Addition of an arch support improves the biomechanical effect of a laterally wedged insole. Gait Posture 2009, 29:208-213.

15. Baker K, Goggins J, Xie H, Szumowski K, LaValley M, Hunter DJ, Felson DT: A randomized crossover trial of a wedged insole for treatment of knee osteoarthritis. Arthritis Rheum 2007, 56:1198-1203.

16. Shelburne KB, Torry MR, Steadman JR, Pandy MG: Effects of foot orthoses and valgus bracing on the knee adduction moment and medial joint load during gait. Clin Biomech (Bristol, Avon) 2008, 23:814-821.

17. Hinman RS, Bowles KA, Payne C, Bennell KL: Effect of length on laterallywedged insoles in knee osteoarthritis. Arthritis Rheum 2008, 59:144-147.

18. Hinman RS, Payne C, Metcalf BR, Wrigley TV, Bennell KL: Lateral wedges in knee osteoarthritis: What are their immediate clinical and biomechanical effects and can these predict a three-month clinical outcome? Arthritis Rheum 2008, 59:408-415.

19. Donatelli R: Abnormal biomechanics of the foot and ankle. J Orthop Sports Phys Ther 1987, 9:11-16.

20. Tiberio D: The effect of excessive subtalar joint pronation on patellofemoral mechanics: a theoretical model. J Orthop Sports Phys Ther 1987, 9:160-165.

21. Guichet JM, Javed A, Russell J, Saleh M: Effect of the foot on the mechanical alignment of the lower limbs. Clin Orthop Relat Res 2003, 193-201.

22. Kerrigan DC, Lelas JL, Goggins J, Merriman GJ, Kaplan RJ, Felson DT: Effectiveness of a lateral-wedge insole on knee varus torque in patients with knee osteoarthritis. Arch Phys Med Rehabil 2002, 83:889-893.
23. Rodrigues PT, Ferreira AF, Pereira RM, Bonfa E, Borba EF, Fuller R: Effectiveness of medial-wedge insole treatment for valgus knee osteoarthritis. Arthritis Rheum 2008, 59:603-608.

24. Reilly K, Barker K, Shamley D, Sandall S: Influence of foot characteristics on the site of lower limb osteoarthritis. Foot Ankle Int 2006, 27:206-211.

25. Reilly K, Barker K, Shamley D, Newman M, Oskrochi GR, Sandall S: The role of foot and ankle assessment of patients with lower limb osteoarthritis. Physiotherapy 2009, 95:164-169.

26. Wrobel JS, Armstrong DG: Reliability and validity of current physical examination techniques of the foot and ankle. J Am Podiatr Med Assoc 2008, 98:197-206.

27. Barton CJ, Bonanno D, Levinger P, Menz HB: Foot and ankle characteristics in patellofemoral pain syndrome: a case control and reliability study. J Orthop Sports Phys Ther 2010, 40:286-296.

28. Vinicombe A, Raspovic A, Menz HB: Reliability of navicular displacement measurement as a clinical indicator of foot posture. J Am Podiatr Med Assoc 2001, 91:262-268.

29. Redmond AC, Crosbie J, Ouvrier RA: Development and validation of a novel rating system for scoring standing foot posture: the Foot Posture Index. Clin Biomech (Bristol, Avon) 2006, 21:89-98.

30. Picciano A, Rowlands MS, Worrell T: Reliability of open and closed kinetic chain subtalar joint neutral positions and navicular drop test. J Orthop Sports Phys Ther 1993, 18:553-558.

31. Brandt K, Fife R, Braunstein E: Radiographic grading of the severity of knee osteoarthritis: relation of the Kellgren and Lawrence grade to a grade based on joint space narrowing and correlation with arthroscopic evidence of articular cartilage degeneration. Arthritis Rheum 1989, 32:1584-1591.

32. Keenan AM, Redmond AC, Horton M, Conaghan PG, Tennant A: The Foot Posture Index: Rasch analysis of a novel, foot-specific outcome measure. Arch Phys Med Rehabil 2007, 88:88-93.

33. Menz HB: Alternative techniques for the clinical assessment of foot pronation. J Am Podiatr Med Assoc 1998, 88:119-129.

34. Williams DS, McClay IS: Measurements used to characterize the foot and the medial longitudinal arch: reliability and validity. Phys Ther 2000, 80:864-871.

35. Cavanagh PR, Rodgers MM: The arch index: a useful measure from footprints. J Biomech 1987, 20:547-551.

36. Portney LG, Watkins MP: Foundation of Clinical Research: Application to Practice East Norwalk, Connecticut:: Appleton \& Lange; 1993.

37. Portney LG, Watkins MP: Foundations of Clinical Research: Applocation to Practice. 2 edition. Upper Saddle River, NJ: Prentice-Hall; 2002.

38. Thalheimer W, Cook S: How to calculate effect sizes from research articles: A simplified methodology. 2002 [http://docs.docstoc.com/pdf/ 5121652/6af10ee0-3d03-46ac-bd77-6a17477830e7.pdf].

39. McWilliams DF, Doherty S, Maciewicz RA, Muir KR, Zhang W, Doherty M: Self-reported knee and foot alignments in early adult life and risk of osteoarthritis. Arthritis Care Res (Hoboken) 2010, 62:489-495.

40. Sharma L, Song J, Dunlop D, Felson D, Lewis CE, Segal N, Torner J, Cooke TD, Hietpas J, Lynch J, Nevitt M: Varus and valgus alignment and incident and progressive knee osteoarthritis. Ann Rheum Dis 2010, 69:1940-1945.

41. Riegger-Krugh C, Keysor JJ: Skeletal malalignment of the lower quarter: correlated and compensatory motions and postures. J Orthop Sports Phys Ther 1996, 23:164-170.

42. Van Gheluwe B, Kirby KA, Hagman F: Effects of simulated genu valgum and genu varum on ground reaction forces and subtalar joint function during gait. J Am Podiatr Med Assoc 2005, 95:531-541.

43. Desai SS, Shetty GM, Song HR, Lee SH, Kim TY, Hur CY: Effect of foot deformity on conventional mechanical axis deviation and ground mechanical axis deviation during single leg stance and two leg stance in genu varum. Knee 2007, 14:452-457.

44. Nester CJ, van der Linden ML, Bowker P: Effect of foot orthoses on the kinematics and kinetics of normal walking gait. Gait Posture 2003, 17:180-187.

45. Kakihana W, Akai M, Nakazawa K, Takashima T, Naito K, Torii S: Effects of laterally wedged insoles on knee and subtalar joint moments. Arch Phys Med Rehabil 2005, 86:1465-1471.

46. Hinman RS, Payne C, Metcalf BR, Wrigley TV, Bennell KL: Lateral wedges in knee osteoarthritis: what are their immediate clinical and biomechanical 
effects and can these predict a three-month clinical outcome? Arthritis Rheum 2008, 59:408-415.

47. Kakihana W, Akai M, Nakazawa K, Naito K, Torii S: Inconsistent knee varus moment reduction caused by a lateral wedge in knee osteoarthritis. Am J Phys Med Rehabil 2007, 86:446-454.

48. Menz HB, Munteanu SE: Validity of 3 clinical techniques for the measurement of static foot posture in older people. J Orthop Sports Phys Ther 2005, 35:479-486.

49. Evans AM, Copper AW, Scharfbillig RW, Scutter SD, Williams MT: Reliability of the foot posture index and traditional measures of foot position. J Am Podiatr Med Assoc 2003, 93:203-213.

50. Pierrynowski MR, Smith SB: Effect of patient position on the consistency of placing the rearfoot at subtalar neutral. J Am Podiatr Med Assoc 1997, 87:399-406.

51. Wearing SC, Hills AP, Byrne NM, Hennig EM, McDonald M: The arch index: a measure of flat or fat feet? Foot Ankle Int 2004, 25:575-581.

doi:10.1186/1757-1146-3-29

Cite this article as: Levinger et al:: Foot posture in people with medial compartment knee osteoarthritis. Journal of Foot and Ankle Research 2010 3:29.

\section{Submit your next manuscript to BioMed Central} and take full advantage of:

- Convenient online submission

- Thorough peer review

- No space constraints or color figure charges

- Immediate publication on acceptance

- Inclusion in PubMed, CAS, Scopus and Google Scholar

- Research which is freely available for redistribution

Submit your manuscript at www.biomedcentral.com/submit 